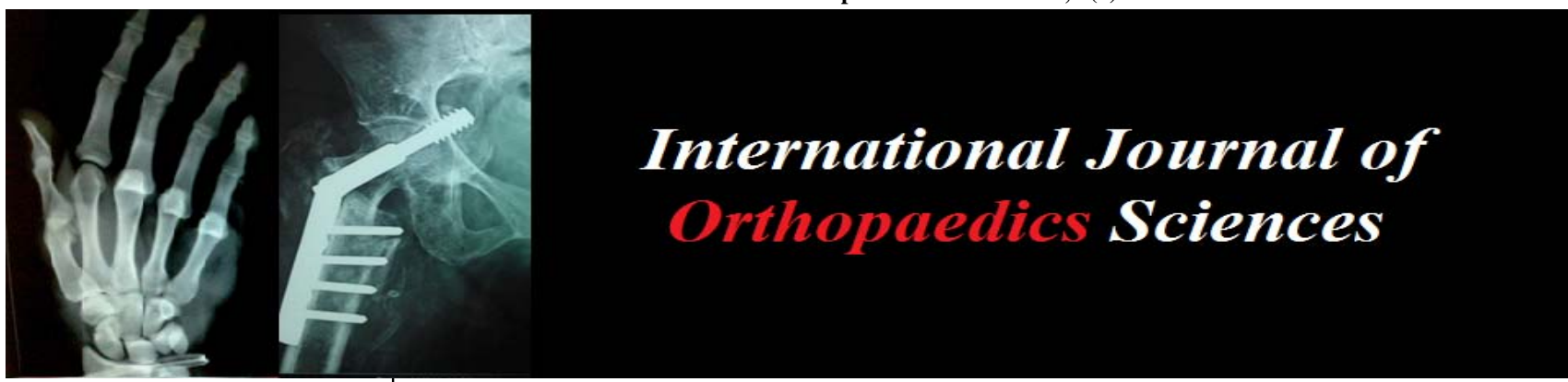

ISSN: $2395-1958$

IJOS 2019; 5(2): 678-680

(C) 2019 IJOS

www.orthopaper.com

Received: 15-02-2019

Accepted: 19-03-2019

Dr. Bandela Manoranjan

Junior Resident,

Dept of Orthopaedics,

MIMS Vizianagaram,

Andhra Pradesh, India

Dr. Yammala Satish Kumar Assistant Professor,

Dept of Orthopaedics,

MIMS Vizianagaram,

Andhra Pradesh, India

Dr. Randhi Rama Kartheek Junior Resident,

Dept of Orthopaedics,

MIMS Vizianagaram,

Andhra Pradesh, India

Dr. Rayapureddy Sridhar

Junior Resident,

Dept of Orthopaedics,

MIMS Vizianagaram,

Andhra Pradesh, India

Dr. Konda Naga Anwesh

Junior Resident,

Dept of Orthopaedics,

MIMS Vizianagaram,

Andhra Pradesh, India

Correspondence

Dr. Bandela Manoranjan

Junior Resident,

Dept of Orthopaedics,

MIMS Vizianagaram,

Andhra Pradesh, India

\section{Metallosis an uncommon complication of total knee joint replacements: A case report}

\author{
Dr. Bandela Manoranjan, Dr. Yammala Satish Kumar, Dr. Randhi Rama \\ Kartheek, Dr. Rayapureddy Sridhar and Dr. Konda Naga Anwesh
}

DOI: https://doi.org/10.22271/ortho.2019.v5.i2j.72

Abstract

The author reports on a patient a case of severe metallosis in total knee replacement. Wear of total knee replacement continues to be a significant factor influencing the clinical longevity of implants. Here we present a case of a 63-year-old female who had undergone total knee arthroplasty 15 years ago and developed implant loosening associated with severe metallosis and varus instability the metallic debris leads to a massive local and systemic release of cytokines. This patient was subjected to revision surgery consisting of thorough debridement and primary implant replacement by a hinged endoprosthesis.

Keywords: Metallosis synovitis Total knee arthroplasty Hinge prosthesis

\section{Introduction}

Metallosis is a rare complication of TKR, and results from the deposition of metallic debris on periprosthetic soft tissues, inducing chronic synovitis and implant loosening. As it a rare complication in total knee prosthesis models without a metal-backed patellar component, we present a case of severe metallosis in a patient who had undergone TKR to treat osteoarthritis of the knee. Metallosis involving the knee joint most often results from metal-on-metal contact late in the life of a failing implant following polyethylene wear. Early diagnosis of metallosis can prevent further damaging to the joint and systemic complications.

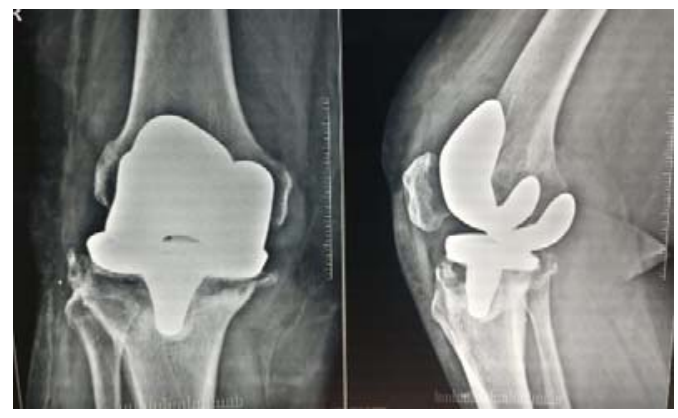

Fig 1: Pre-operative assessment $X$-ray

\section{Case Report}

A 63-year-old female who had undergone bilateral TKR 15 years ago at another institution to treat primary osteoarthritis of the both the knees was admitted to our institution for progressive right knee pain, gait disability, and development of a mass on the anteromedial aspect of the right knee. Physical examination of the ligamentous structures of the right knee showed varus instability. The patient's knee had full active and passive range of motion. There were no signs of swelling, flushing, increased temperature on the right knee. Anteroposterior and lateral radiographs showed signs of osteolysis of the femur and tibia, and signs of loosening of the femoral and tibial components. Laboratory findings from complete blood picture, CRP, and ESR were normal which shows no signs of infection. There are no records of intraoperative 
complications. In our experience, most TKRs carried out with this implant have approximately 10 years of survivorship. Moreover, there are no cases of metallosis associated with this implant documented in our institution

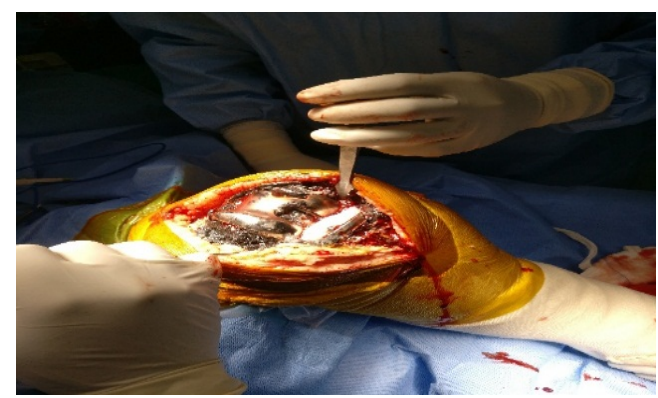

Fig 2: Intraoperative showing Metallosis

During the first 4 years after the primary arthroplasty the patient was being monitored annually, when she herself discontinued treatment because she was asymptomatic from the operated right knee. When symptoms initiated, 3 years later, the patient complained of intermittent pain that was treated with analgesics, nonsteroidal anti-inflammatory drugs. Approximately 3 years later (10 years after surgery), she progressed to gait disability and decided to re-sume consultation. At this time she approached our institution.

In our institution, following clinical and radiographic evaluation, the diagnosis of metallosis-induced prosthesis loosening thus, a hinged endoprosthesis was planned for TKR revision surgery 20 days after the patient's admission. Since clinical and laboratory findings were not suggestive of chronic infection, no preoperative joint aspiration was performed. Instead, one-stage TKR revision surgery was planned and cefuroxime was administered with a dosage of $1.5 \mathrm{~g}$ intravenously $30 \mathrm{~min}$ before skin incision and was maintained until the results of the microbiological culture. Intraoperatively, severe black staining of the synovial fluid and periprosthetic soft tissues were noted. Additionally, extensive destruction of the tibial component of the medial compartment and polyethylene wear were observed. During the surgical procedure debridement of abnormal tissue was conducted and samples of bone and soft tissue for anatomopathological and microbiological examination were collected. no postoperative signs of infection in sequential physical and laboratory (complete blood count, C-reactive protein and erythrocyte sedimentation rate) examinations The sample of soft tissue sent to histopathological examination was blackish areas and had a softened consistency. Under direct microscopic visuali-zation, giant cells with a pattern of foreign body reaction (nonspecific chronic synovitis) and metallic impregnation were observed, which confirmed the hypothesis of metallosis.

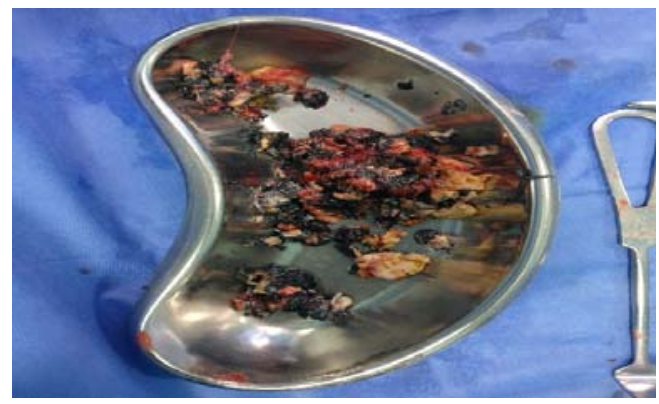

Fig 3: black stained soft tissue

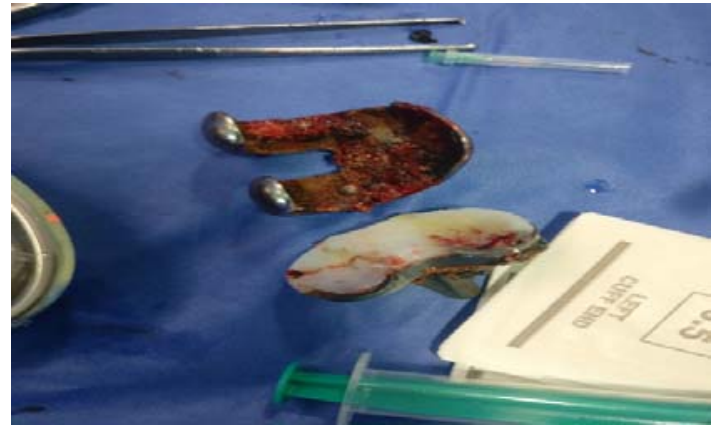

Fig 4: Removal of loosened Implant

She was discharged on the tenth postoperative day with oral antibiotic therapy to treat the subclinical infection. She was closely monitored monthly with physical, laboratory, and radio-graphic examinations. Currently, 6 months after total knee arthroplasty revision, the patient is asymptomatic, has a full range of motion. Current radio-graphs demonstrate a well-positioned prosthesis with no signs of implant loosening, fracture or osteolysis. Immediate postoperative radiographs are similar to current radiographs, implying an excellent outcome. The study was approved by the ethics committee of our institution and informed consent was obtained.

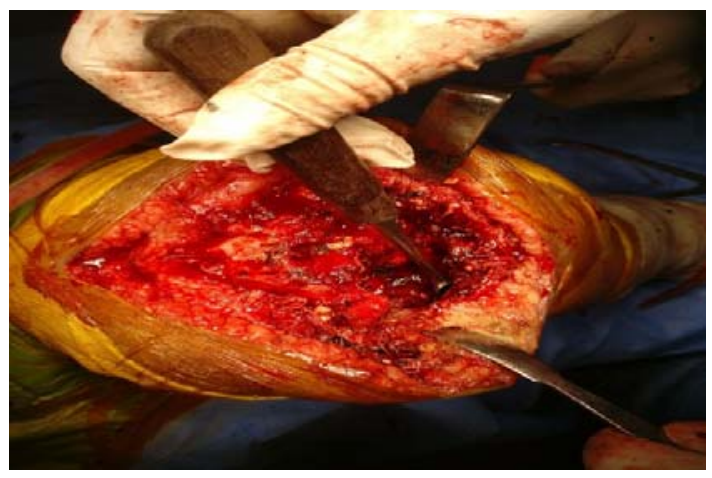

Fig 5: debridement of abnormal tissue

\section{Discussion}

In this case report, a 63-year-old female developed metallosis 15 years after she had undergone a TKR. This complication is not exclusive to TKR and may occur following hip (highest incidence), shoulder, elbow, and wrist arthroplasties ${ }^{1}$. Metallosis is believed to be mechanically-induced, resulting from abrasion between the metallic com-ponents; or between the metallic component and the polyethylene surface. Metallic impregnation can also make the synovial fluid and periprosthetic soft tissues blackish.

This patient suffered from chronic synovitis induced by metallosis and subclinical infection. This association is uncommon, with a reported incidence of $9 \%$ in a retrospective study ${ }^{[3]}$. Both metallosis and infection decrease prosthesis survivorship as they induce and accelerate periprosthetic osteolysis [1,4] There-after, chronic synovitis worked as a positive feedback mech-anism, leading to greater joint destruction, which increased knee instability and excessive wear of the medial compart-ment. Most likely, the patient had chronic synovitis of the knee for at least 5 years without any clinical or radiographic signs.

Progressive joint destruction triggered by metallosis should be avoided by debridement consisting of synovectomy and resection of the inflammatory tissue, and by performing a revision arthroplasty ${ }^{[1,2]}$. Competent and functional collateral 
ligaments are prerequisites when performing a conventional TKR. addition to the massive metallosis-induced osteolysis, the patient's knee had severe deficiency of the lateral ligaments; therefore, the revision surgery was performed with a hinged endoprosthesis, which possesses intrinsic lateral and medial stability ${ }^{[5,6]}$.

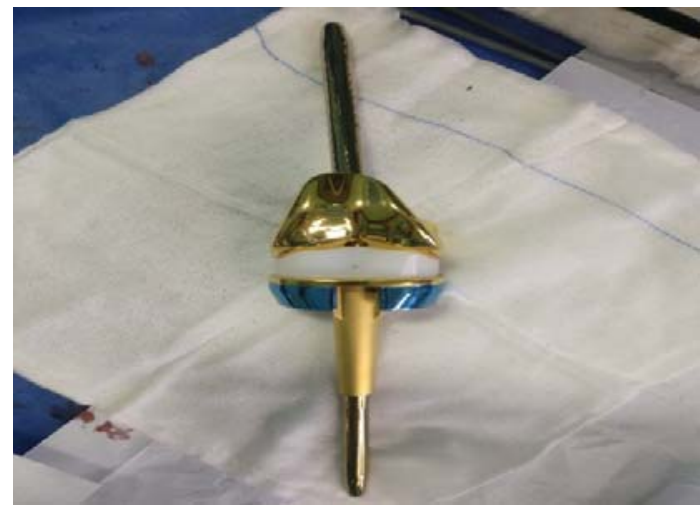

Fig 6: Hinge Prosthesis for revision TKA

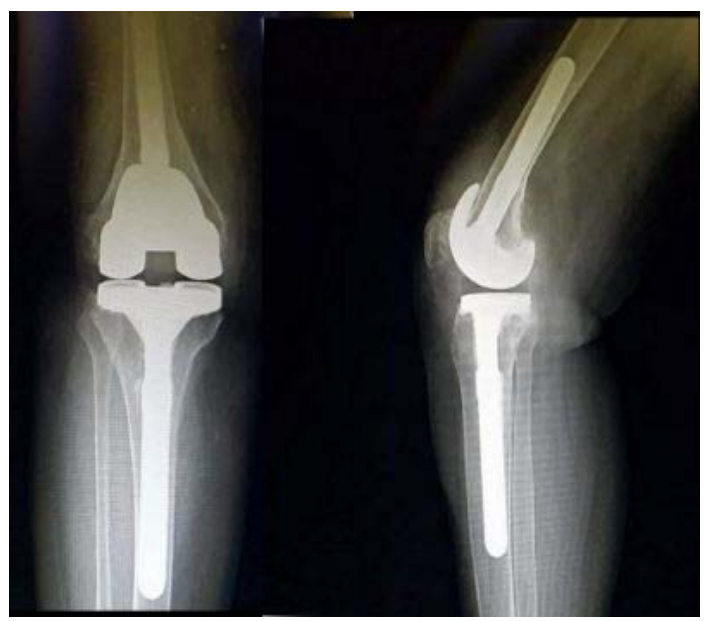

Fig 7: Post-operative X-ray

\section{Conclusion}

This case report shows the importance of the long-term follow-up of patients subjected to TKR in the early diagnosis of its complications. Metallosis diagnosed early can be treated with debridement of abnormal tissue and revision surgery, thus preventing extensive damage to the patient's joint.

\section{References}

1. Romesburg JW, Wasserman PL, Schoppe CH. Metallosis and metal-induced synovitis following total knee arthroplasty: review of radiographic and CT findings. J Radiol Case Rep. 2010; 4(9):7-17.

2. Chew FS, Ramsdell MG, Keel SB. Metallosis after total knee re-placement. Am J Roentgenol. 1998; 170(6):1556.

3. Forster MC. Survival analysis of primary cemented total knee arthroplasty: which designs last? J Arthroplasty. 2003; 18(3):265-70.

4. Sanchis-Alfonso V. Severe metallosis after unicompartmental knee arthroplasty. Knee Surg Sports Traumatol Arthrosc. 2007; 15(4):361-4.

5. Hernández-Vaquero D, Sandoval-García MA. Hinged total knee arthroplasty in the presence of ligamentous deficiency. Clin Orthop Relat Res. 2010; 468(5):1248-53.

6. Joshi $\mathrm{N}$, Navarro-Quilis. Is there a place for rotating- hinge arthroplasty in knee revision surgery for aseptic loosening? J Arthroplasty. 2008; 23(8):1204-11. 